\title{
On a discrete-time risk model with delayed claims and dividends
}

\author{
Kam Chuen Yuen*, Jinzhu $\mathrm{Li}^{\dagger}, \quad$ Rong $\mathrm{Wu}^{\dagger}$ \\ *Department of Statistics and Actuarial Science, \\ The University of Hong Kong, Hong Kong \\ †School of Mathematical Sciences and LPMC, \\ Nankai University Tianjin 300071, China
}

\begin{abstract}
In this paper, we study the discounted free Gerber-Shiu function for the compound binomial risk model with by-claims and randomized dividend policy. Specifically, explicit expression for the discounted free Gerber-Shiu function is obtained. This result allows us to derive formulae for some useful insurance quantities, including the ruin probability, the probability function of the deficit at ruin, the joint probability function of the surplus immediately before ruin and the deficit at ruin, and the probability function of the claim causing ruin.
\end{abstract}

Keywords: By-claim; Claim causing ruin; Compound binomial risk model; Deficit at ruin; Gerber-Shiu function; Main claim; Randomized dividend; Ruin probability; Surplus immediately before ruin

AMS subject classifications: Primary 91B30; Secondary 60H30.

\section{Introduction}

Research concerning the compound binomial risk model with i.i.d. claims began from Gerber (1988), in which he derived formulae for the ruin probability, the distribution of the deficit at ruin, and the distribution of the surplus immediately before ruin when the initial surplus is zero. Since then, this topic has been extensively studied by many authors including Shiu (1989), Willmot (1993), and Pavlova and Willmot (2004). Since the independence assumption imposed on claims in the classical model is not realistic, generalizations of the model with various kinds of dependency were considered in the literature. Among others, Yuen and Guo (2001) considered the compound binomial model with the so-called by-claims, that is, every (main) claim causes a by-claim whose time of occurrence may be delayed to the next time period. In their paper, they stated the practical background of the model and obtained the recursive formula for the finite time ruin probability as well as the explicit expressions for ultimate ruin probabilities in special cases. Recently, Xiao and Guo (2007) studied the by-claim model further, and derived the recursive formula for the joint probability function of the surplus immediately before ruin and the deficit at ruin. For continuoustime risk models with delayed claims, see, for example, Yuen, Guo and Ng (2005). Instead of

\footnotetext{
*Corresponding author. Email: kcyuen@hku.hk
} 
introducing dependency to the claims, Tan and Yang (2006) modified the compound binomial model in the way that the insurer may pay dividend with a certain probability if the surplus is greater than or equal to a fixed nonnegative integer. They derived the recursive formula and asymptotic estimate for the discounted free Gerber-Shiu function for their model with the so-called randomized dividend policy.

In the present paper, we consider the compound binomial risk model with by-claims under the randomized dividend policy. Besides adopting the by-claim set-up in Yuen and Guo (2001), we assume that the insurer may pay a randomized dividend of 1 with a certain probability at the beginning of every time period until ruin occurs. Different from all the above-mentioned references in which explicit formulae are derived only in the special case of zero initial surplus, we obtain explicit formula for the discount free Gerber-Shiu function for all nonnegative initial surplus. Furthermore, based on the derived formula, we study some useful insurance quantities including the ruin probability, the probability function of the deficit at ruin, the joint probability function of the surplus immediately before ruin and the deficit at ruin, and the probability function of the claim causing ruin.

The rest of this paper is organized as follows. In Section 2, we introduce the compound binomial risk model with by-claims and randomized dividends. In Section 3, explicit formula for the discounted free Gerber-Shiu function is derived. In Section 4, we study some useful insurance quantities using the formula obtained in Section 3.

\section{The model}

The discrete-time risk model considered in this paper is

$$
U_{t}=u+t-\sum_{i=1}^{t} \zeta_{i} \mathbf{1}_{\left(U_{i-1} \geq 0\right)}-\sum_{i=1}^{t} Z_{i}, \quad t=0,1,2, \ldots,
$$

where

$$
\begin{aligned}
Z_{1} & =\xi_{1}\left(X_{1}+\eta_{1} Y_{1}\right) \\
Z_{i} & =\xi_{i}\left(X_{i}+\eta_{i} Y_{i}\right)+\xi_{i-1}\left(1-\eta_{i-1}\right) Y_{i-1}, \quad i=2,3, \ldots
\end{aligned}
$$

Here, the nonnegative integers $u$ and $t$ are the initial surplus and time period, respectively. A premium of 1 is received at the beginning of every period, and $\mathbf{1}_{A}$ is the indicator function of an event $A$. Main claim amounts $X_{i}$ and by-claim amounts $Y_{i}$ are positive integer-valued i.i.d. random variables with probability functions, $P(X=k)=f_{k}$ and $P(Y=l)=g_{l}$ for $k, l=1,2, \ldots$, and means $E(X)=\mu_{X}$ and $E(Y)=\mu_{Y}$. Furthermore, $\zeta_{i}, \xi_{i}$, and $\eta_{i}$ are indicator random variables. Specifically, $\zeta_{i}=1$ with probability $\alpha$ indicates that a dividend of 1 is paid at the beginning of the $i$ th period if ruin does not occur, and $\zeta_{i}=0$ with probability $1-\alpha$ indicates that no dividend is paid at the beginning of the $i$ th period; $\xi_{i}=1$ with probability $p$ indicates that there is a main claim in the $i$ th period (main claim amount is payable at the end of the $i$ th period), and $\xi_{i}=0$ with probability $1-p$ indicates that there is no main claim in the $i$ th period; $\eta_{i}=1$ with probability $\theta$ indicates that the by-claim induced by the main claim in the $i$ th period is paid at the the end of the $i$ th period (both the main claim and its induced by-claim are payable at the end of the $i$ th period), and $\eta_{i}=0$ with probability $1-\theta$ indicates that the by-claim induced by the main claim 
in the $i$ th period is paid a period later. As a result, $Z_{i}$ defined in (2.2) is the total of the main claim and by-claim amounts payable at the end of the $i$ th period. As usual, it is assumed that $\left\{\zeta_{i}\right\}$, $\left\{\xi_{i}\right\},\left\{\eta_{i}\right\},\left\{X_{i}\right\}$ and $\left\{Y_{i}\right\}$ are independent. On the other hand, it is clear that every two successive claims $Z_{i-1}$ and $Z_{i}$ are correlated. Additionally, we set $\sum_{a}^{b}=0$ if $a>b$, and assume that the positive safety loading condition $1-\alpha-p\left(\mu_{X}+\mu_{Y}\right)>0$ holds to guarantee that ruin does not occur with probability 1.

In risk model (2.1), if $\alpha=0$, the model reduces to the by-claim model without dividends in Yuen and Guo (2001), while if $\theta=1$, by-claims always occur together with their corresponding main claims and hence the model collapses to that in Tan and Yang (2006) with zero dividend-bound and claims $Z_{i}=X_{i}+Y_{i}$. Certainly, if $\theta=1$ and $\alpha=0$, model (2.1) degenerates to the classical compound binomial model which has been studied extensively.

Gerber and Shiu (1998) introduced the expected discounted penalty function with respect to the time of ruin, the surplus immediately before ruin and the deficit at ruin, which has proved to be a powerful analytical tool in risk theory. Let $\tau=\inf \left\{t ; U_{t}<0\right\}$ be the ruin time of model (2.1), with $\tau=\infty$ if ruin does not occur. Then, given $\tau<\infty, U_{\tau-}$ is the surplus immediately before ruin, and $\left|U_{\tau}\right|$ is the deficit at ruin. For any nonnegative bounded function $\omega\left(v_{1}, v_{2}\right)$ and any discount factor $0<v \leq 1$, the Gerber-Shiu expected discounted penalty function for model (2.1) is defined as

$$
m_{v}(u)=E\left(v^{\tau} \omega\left(U_{\tau-},\left|U_{\tau}\right|\right) \mathbf{1}_{(\tau<\infty)} \mid U_{0}=u\right) .
$$

Here, we only consider the case with $v=1$ and study the discounted free Gerber-Shiu function

$$
m(u)=E\left(\omega\left(U_{\tau-},\left|U_{\tau}\right|\right) \mathbf{1}_{(\tau<\infty)} \mid U_{0}=u\right)
$$

\section{$3 \quad$ Explicit expression for $m(u)$}

Following Yuen and Guo (2001), we define an auxiliary process

$$
U_{t}^{\prime}=u+t-\sum_{i=1}^{t} \zeta_{i} \mathbf{1}_{\left(U_{i-1}^{\prime} \geq 0\right)}-\sum_{i=1}^{t} Z_{i}-Y^{\prime} \mathbf{1}_{(t \geq 1)},
$$

where $Y^{\prime}$ has the same probability probability function as $Y_{i}^{\prime}$ 's. Denote by $m^{\prime}(u)$ the discounted free Gerber-Shiu function for $U_{t}^{\prime}$. We will see that the process $U_{t}^{\prime}$ plays an important role in deriving our main results.

For model (2.1), there are several cases at time 1 according to whether a main claim occurs or not, whether the associated by-claim occurs simultaneously or occurs in the next period, whether the dividend is paid or not, and whether ruin occurs or not. Taking into account all these cases and using the law of total probability, we have 


$$
\begin{aligned}
m(u)= & q(1-\alpha) m(u+1)+q \alpha m(u)+p \theta(1-\alpha) \sum_{k+l=2}^{u+1} m(u+1-k-l) f_{k} g_{l} \\
& +p \theta(1-\alpha) \sum_{k+l=u+2}^{\infty} \omega(u+1, k+l-u-1) f_{k} g_{l}+p \theta \alpha \sum_{k+l=2}^{u} m(u-k-l) f_{k} g_{l} \\
& +p \theta \alpha \sum_{k+l=u+1}^{\infty} \omega(u, k+l-u) f_{k} g_{l}+p(1-\theta)(1-\alpha) \sum_{k=1}^{u+1} m^{\prime}(u+1-k) f_{k} \\
& +p(1-\theta)(1-\alpha) \sum_{k=u+2}^{\infty} \omega(u+1, k-u-1) f_{k}+p(1-\theta) \alpha \sum_{k=1}^{u} m^{\prime}(u-k) f_{k} \\
& +p(1-\theta) \alpha \sum_{k=u+1}^{\infty} \omega(u, k-u) f_{k} .
\end{aligned}
$$

Similarly, for model (3.1), we obtain

$$
\begin{aligned}
m^{\prime}(u)= & q(1-\alpha) \sum_{l=1}^{u+1} m(u+1-l) g_{l}+q(1-\alpha) \sum_{l=u+2}^{\infty} \omega(u+1, l-u-1) g_{l}+q \alpha \sum_{l=1}^{u} m(u-l) g_{l} \\
& +q \alpha \sum_{l=u+1}^{\infty} \omega(u, l-u) g_{l}+p \theta(1-\alpha) \sum_{l+k+n=3}^{u+1} m(u+1-l-k-n) g_{l} f_{k} g_{n} \\
& +p \theta(1-\alpha) \sum_{l+k+n=u+2}^{\infty} \omega(u+1, l+k+n-u-1) g_{l} f_{k} g_{n}+p \theta \alpha \sum_{l+k+n=3}^{u} m(u-l-k-n) g_{l} f_{k} g_{n} \\
& +p \theta \alpha \sum_{l+k+n=u+1}^{\infty} \omega(u, l+k+n-u) g_{l} f_{k} g_{n}+p(1-\theta)(1-\alpha) \sum_{k+l=2}^{u+1} m^{\prime}(u+1-k-l) f_{k} g_{l} \\
& +p(1-\theta)(1-\alpha) \sum_{k+l=u+2}^{\infty} \omega(u+1, k+l-u-1) f_{k} g_{l}+p(1-\theta) \alpha \sum_{k+l=2}^{u} m^{\prime}(u-k-l) f_{k} g_{l} \\
& +p(1-\theta) \alpha \sum_{k+l=u+1}^{\infty} \omega(u, k+l-u) f_{k} g_{l} .
\end{aligned}
$$

Let

$$
\begin{gathered}
W_{1}(u)=\sum_{k+l=u+1}^{\infty} \omega(u, k+l-u) f_{k} g_{l}, \\
W_{2}(u)=\sum_{k=u+1}^{\infty} \omega(u, k-u) f_{k}, \\
W_{3}(u)=\sum_{l=u+1}^{\infty} \omega(u, l-u) g_{l}, \\
W_{4}(u)=\sum_{l+k+n=u+1}^{\infty} \omega(u, l+k+n-u) g_{l} f_{k} g_{n},
\end{gathered}
$$

and denote by $f_{1} * f_{2}$ the convolution of $f_{1}$ and $f_{2}$ with $f_{1} * f_{2}(u)=\sum_{i=0}^{u} f_{1}(u-i) f_{2}(i)$. Then, (3.2) and (3.3) can be simplified as 


$$
\begin{aligned}
(1-q \alpha) m(u)= & q(1-\alpha) m(u+1)+p \theta(1-\alpha) m * f * g(u+1)+p \theta \alpha m * f * g(u) \\
& +p(1-\theta)(1-\alpha) m^{\prime} * f(u+1)+p(1-\theta) \alpha m^{\prime} * f(u)+p \theta(1-\alpha) W_{1}(u+1) \\
& +p \theta \alpha W_{1}(u)+p(1-\theta)(1-\alpha) W_{2}(u+1)+p(1-\theta) \alpha W_{2}(u),
\end{aligned}
$$

and

$$
\begin{aligned}
m^{\prime}(u)= & q(1-\alpha) m * g(u+1)+q \alpha m * g(u)+p \theta(1-\alpha) m * f * g^{* 2}(u+1)+p \theta \alpha m * f * g^{* 2}(u) \\
& +p(1-\theta)(1-\alpha) m^{\prime} * f * g(u+1)+p(1-\theta) \alpha m^{\prime} * f * g(u)+p(1-\theta)(1-\alpha) W_{1}(u+1) \\
& +p(1-\theta) \alpha W_{1}(u)+q(1-\alpha) W_{3}(u+1)+q \alpha W_{3}(u)+p \theta(1-\alpha) W_{4}(u+1)+p \theta \alpha W_{4}(u) .
\end{aligned}
$$

In the rest of this paper, we put a tilde on top of a function to denote its generating function, for example $\widetilde{f}(z)=\sum_{i=0}^{\infty} z^{i} f_{i}, \widetilde{W_{1}}(z)=\sum_{i=0}^{\infty} z^{i} W_{1}(i)$, and so on. Multiplying (3.8) and (3.9) by $z^{u+1}$ and summing over $u$ from 0 to $\infty$, we obtain

$$
\begin{aligned}
& {[(1-q \alpha) z-q(1-\alpha)-p \theta(1-\alpha) \widetilde{f}(z) \widetilde{g}(z)-p \theta \alpha z \widetilde{f}(z) \widetilde{g}(z)] \widetilde{m}(z) } \\
= & {[p(1-\theta)(1-\alpha) \widetilde{f}(z)+p(1-\theta) \alpha z \widetilde{f}(z)] \widetilde{m^{\prime}}(z)+[p \theta(1-\alpha)+p \theta \alpha z] \widetilde{W}_{1}(z) } \\
& +[p(1-\theta)(1-\alpha)+p(1-\theta) \alpha z] \widetilde{W}_{2}(z)-q(1-\alpha) m(0)-p \theta(1-\alpha) W_{1}(0) \\
& -p(1-\theta)(1-\alpha) W_{2}(0),
\end{aligned}
$$

and

$$
\begin{aligned}
& {\left[q(1-\alpha) \widetilde{g}(z)+q \alpha z \widetilde{g}(z)+p \theta(1-\alpha) \widetilde{f}(z) \widetilde{g}^{2}(z)+p \theta \alpha z \widetilde{f}(z) \widetilde{g}^{2}(z)\right] \widetilde{m}(z) } \\
= & {[z-p(1-\theta)(1-\alpha) \widetilde{f}(z) \widetilde{g}(z)-p(1-\theta) \alpha z \widetilde{f}(z) \widetilde{g}(z)] \widetilde{m^{\prime}}(z)-[q(1-\alpha)+q \alpha z] \widetilde{W}_{3}(z) } \\
& -[p(1-\theta)(1-\alpha)+p(1-\theta) \alpha z] \widetilde{W}_{1}(z)-[p \theta(1-\alpha)+p \theta \alpha z] \widetilde{W}_{4}(z) \\
& +p(1-\theta)(1-\alpha) W_{1}(0)+q(1-\alpha) W_{3}(0)+p \theta(1-\alpha) W_{4}(0) .
\end{aligned}
$$

Then, it follows from (3.10) and (3.11) that

$$
\begin{aligned}
& {[(1-q \alpha) z-p \alpha z \widetilde{f}(z) \widetilde{g}(z)-q(1-\alpha)-p(1-\alpha) \widetilde{f}(z) \widetilde{g}(z)] \widetilde{m}(z) } \\
= & {\left[\left(p \theta \alpha-p^{2} \theta(1-\theta) \alpha^{2} \widetilde{f}(z) \widetilde{g}(z)+p^{2}(1-\theta)^{2} \alpha^{2} \widetilde{f}(z)\right) z+\left(p \theta(1-\alpha)-2 p^{2} \theta(1-\theta) \alpha(1-\alpha) \widetilde{f}(z) \widetilde{g}(z)\right.\right.} \\
& \left.\left.\quad+2 p^{2}(1-\theta)^{2} \alpha(1-\alpha) \widetilde{f}(z)\right)+\left(p^{2}(1-\theta)^{2}(1-\alpha)^{2} \widetilde{f}(z)-p^{2} \theta(1-\theta)(1-\alpha)^{2} \widetilde{f}(z) \widetilde{g}(z)\right) \frac{1}{z}\right] \widetilde{W}_{1}(z) \\
+ & {\left[\left(p(1-\theta) \alpha-p^{2}(1-\theta)^{2} \alpha^{2} \widetilde{f}(z) \widetilde{g}(z)\right) z+\left(p(1-\theta)(1-\alpha)-2 p^{2}(1-\theta)^{2} \alpha(1-\alpha) \widetilde{f}(z) \widetilde{g}(z)\right)\right.} \\
& \left.\quad-p^{2}(1-\theta)^{2}(1-\alpha)^{2} \widetilde{f}(z) \widetilde{g}(z) \frac{1}{z}\right] \widetilde{W}_{2}(z) \\
+ & {\left[p(1-\theta) \alpha^{2} \widetilde{f}(z) z+2 p(1-\theta) \alpha(1-\alpha) \widetilde{f}(z)+p(1-\theta)(1-\alpha)^{2} \widetilde{f}(z) \frac{1}{z}\right]\left(q \widetilde{W}_{3}(z)+p \theta \widetilde{W}_{4}(z)\right) } \\
- & {\left[q(1-\alpha)-p q(1-\theta) \alpha(1-\alpha) \widetilde{f}(z) \widetilde{g}(z)-p q(1-\theta)(1-\alpha)^{2} \widetilde{f}(z) \widetilde{g}(z) \frac{1}{z}\right] m(0) } \\
- & {\left[\left(p \theta(1-\alpha)-p^{2} \theta(1-\theta) \alpha(1-\alpha) \widetilde{f}(z) \widetilde{g}(z)+p^{2}(1-\theta)^{2} \alpha(1-\alpha) \widetilde{f}(z)\right)\right.} \\
& \left.\quad+\left(p^{2}(1-\theta)^{2}(1-\alpha)^{2} \widetilde{f}(z)-p^{2} \theta(1-\theta)(1-\alpha)^{2} \widetilde{f}(z) \widetilde{g}(z)\right) \frac{1}{z}\right] W_{1}(0) \\
- & {\left[\left(p(1-\theta)(1-\alpha)-p^{2}(1-\theta)^{2} \alpha(1-\alpha) \widetilde{f}(z) \widetilde{g}(z)\right)-p^{2}(1-\theta)^{2}(1-\alpha)^{2} \widetilde{f}(z) \widetilde{g}(z) \frac{1}{z}\right] W_{2}(0) } \\
- & {\left[p(1-\theta) \alpha(1-\alpha) \widetilde{f}(z)+p(1-\theta)(1-\alpha)^{2} \widetilde{f}(z) \frac{1}{z}\right]\left(q W_{3}(0)+p \theta W_{4}(0)\right) . }
\end{aligned}
$$


It is easy to see that if we set $z=1$ in (3.12), then the left-hand side of (3.12) becomes 0 and hence

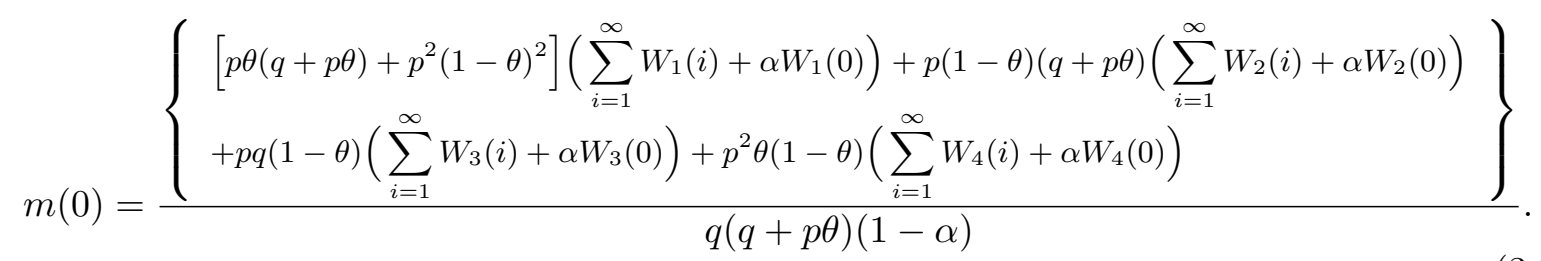

Moreover, by comparing the coefficients of $z^{u+1}$ on both sides of (3.12), one can derive a recursive formula for $m(u)$. For notational convenience, write

$$
h_{i}=P(X+Y=i)=f * g(i), \quad i=2,3, \ldots
$$

Then, after some tedious calculation and rearrangement, we obtain from (3.12) that

$$
\begin{aligned}
& q(1-\alpha) m(u+1) \\
= & (1-q \alpha) m(u)-p \sum_{i=0}^{u-1} m(i)\left[(1-\alpha) h_{u-i+1}+\alpha h_{u-i}\right]-p q(1-\theta)(1-\alpha) m(0)\left[(1-\alpha) h_{u+2}+\alpha h_{u+1}\right] \\
& -p \theta\left[(1-\alpha) W_{1}(u+1)+\alpha W_{1}(u)\right]-p(1-\theta)\left[(1-\alpha) W_{2}(u+1)+\alpha W_{2}(u)\right] \\
& +p^{2}(1-\theta) \alpha\left[\theta W_{1}(0)+(1-\theta) W_{2}(0)\right]\left[(1-\alpha) h_{u+1}+\alpha h_{u}\right] \\
& +p^{2}(1-\theta) \sum_{i=1}^{u}\left[\theta W_{1}(i)+(1-\theta) W_{2}(i)\right]\left[(1-\alpha)^{2} h_{u-i+2}+2 \alpha(1-\alpha) h_{u-i+1}+\alpha^{2} h_{u-i}\right] \\
& -p(1-\theta) \alpha\left[p(1-\theta) W_{1}(0)+q W_{3}(0)+p \theta W_{4}(0)\right]\left[(1-\alpha) f_{u+1}+\alpha f_{u}\right] \\
& -p(1-\theta) \sum_{k=1}^{u+1}\left[p(1-\theta) W_{1}(k)+q W_{3}(k)+p \theta W_{4}(k)\right]\left[(1-\alpha)^{2} f_{u-k+2}+2 \alpha(1-\alpha) f_{u-k+1}+\alpha^{2} f_{u-k}\right] .
\end{aligned}
$$

So, combining (3.13) and (3.15) gives a recursive formula for $m(u)$.

Use capital letters to denote distribution functions, and capital letters with a bar on top to denote survival functions. For example, $F(n)=\sum_{k=1}^{n} f_{k}, \bar{F}(n)=1-F(n)=\sum_{k=n+1}^{\infty} f_{k}$ and so on. For any $t \geq 0$, summing $u$ from $t$ to $\infty$ in (3.15) and rearranging terms yield

$$
\begin{aligned}
0 & =p \sum_{u=t+1}^{\infty} m(u)+(1-q \alpha) m(t)-p \sum_{u=t}^{\infty} \sum_{i=0}^{u-1} m(i)\left[(1-\alpha) h_{u-i+1}+\alpha h_{u-i}\right]-p A(t) \\
& =p \sum_{j=t+1}^{\infty} m(j)+(1-q \alpha) m(t)-p \sum_{j=0}^{\infty} m(j)+p \sum_{j=0}^{t-1} m(j)[(1-\alpha) H(t-j)+\alpha H(t-j-1)]-p A(t) \\
& =q(1-\alpha) m(t)-p \sum_{j=0}^{t-1} m(j)[(1-\alpha) \bar{H}(t-j)+\alpha \bar{H}(t-j-1)]-p A(t),
\end{aligned}
$$


where

$$
\begin{aligned}
A(u)= & q(1-\theta)(1-\alpha) m(0)[(1-\alpha) \bar{H}(u+1)+\alpha \bar{H}(u)] \\
& +\theta \sum_{j=u}^{\infty}\left[(1-\alpha) W_{1}(j+1)+\alpha W_{1}(j)\right]+(1-\theta) \sum_{j=u}^{\infty}\left[(1-\alpha) W_{2}(j+1)+\alpha W_{2}(j)\right] \\
& -p(1-\theta) \alpha\left[\theta W_{1}(0)+(1-\theta) W_{2}(0)\right][(1-\alpha) \bar{H}(u)+\alpha \bar{H}(u-1)] \\
& -p(1-\theta) \sum_{j=u}^{\infty} \sum_{i=1}^{j}\left[\theta W_{1}(i)+(1-\theta) W_{2}(i)\right]\left[(1-\alpha)^{2} h_{j-i+2}+2 \alpha(1-\alpha) h_{j-i+1}+\alpha^{2} h_{j-i}\right] \\
& +(1-\theta) \alpha\left[p(1-\theta) W_{1}(0)+q W_{3}(0)+p \theta W_{4}(0)\right][(1-\alpha) \bar{F}(u)+\alpha \bar{F}(u-1)] \\
& +(1-\theta) \sum_{j=u}^{\infty} \sum_{k=1}^{j+1}\left[p(1-\theta) W_{1}(k)+q W_{3}(k)+p \theta W_{4}(k)\right]\left[(1-\alpha)^{2} f_{j-k+2}+2 \alpha(1-\alpha) f_{j-k+1}+\alpha^{2} f_{j-k}\right] .
\end{aligned}
$$

Note that the second equality in (3.16) is derived by interchanging the order of summation in the third term in the first line of (3.16). Putting $t=u$ in (3.16), we have

$$
q(1-\alpha) m(u)=p \sum_{j=0}^{u-1} m(j)[(1-\alpha) \bar{H}(u-j)+\alpha \bar{H}(u-j-1)]+p A(u) .
$$

Finally, $m(u)$ can be solved explicitly from (3.18) using the technique of generating functions.

Theorem 3.1 For every $u=0,1,2, \ldots$, the discounted free Gerber-Shiu function $m(u)$ for model (2.1) can be expressed explicitly as

$$
m(u)=\frac{p}{1-\alpha-p\left(\mu_{X}+\mu_{Y}\right)} \sum_{j=0}^{u} A(u-j) \sigma(j)=\frac{p \cdot A * \sigma(u)}{1-\alpha-p\left(\mu_{X}+\mu_{Y}\right)},
$$

where

$$
\left\{\begin{array}{l}
\sigma(0)=\frac{\lambda}{1+\lambda}+\frac{\lambda}{1+\lambda} \sum_{n=1}^{\infty}\left(\frac{1}{1+\lambda}\right)^{n} \rho^{* n}(0), \\
\sigma(j)=\frac{\lambda}{1+\lambda} \sum_{n=1}^{\infty}\left(\frac{1}{1+\lambda}\right)^{n} \rho^{* n}(j), \quad j=1,2, \ldots,
\end{array}\right.
$$

with

$$
\lambda=\frac{1-\alpha-p\left(\mu_{X}+\mu_{Y}\right)}{p\left(\alpha+\mu_{X}+\mu_{Y}\right)},
$$

and

$$
\rho(j)=\frac{1}{\alpha+\mu_{X}+\mu_{Y}}[(1-\alpha) \bar{H}(j)+\alpha \bar{H}(j-1)], \quad j=0,1,2, \ldots
$$

Proof. Using (3.21) and (3.22), one can rewrite (3.17) as

$$
\begin{aligned}
m(u) & =\frac{p}{1-q \alpha} \sum_{j=0}^{u} m(u-j)[(1-\alpha) \bar{H}(j)+\alpha \bar{H}(j-1)]+\frac{p}{1-q \alpha} A(u) \\
& =\frac{1}{1+\lambda} \sum_{j=0}^{u} m(u-j) \rho(j)+\frac{1}{1+\lambda} B(u),
\end{aligned}
$$


where $B(u)=A(u) /\left(\alpha+\mu_{X}+\mu_{Y}\right)$. From (3.23), it is easy to see that

$$
\widetilde{m}(z)=\frac{\widetilde{B}(z)}{1+\lambda-\widetilde{\rho}(z)} .
$$

On the other hand, it follows from (3.20) that

$$
\tilde{\sigma}(z)=\sum_{j=0}^{\infty} z^{j} \sigma(j)=\frac{\lambda}{1+\lambda-\widetilde{\rho}(z)} .
$$

Then, substituting (3.25) into (3.24) leads to

$$
\widetilde{m}(z)=\frac{1}{\lambda} \widetilde{\sigma}(z) \widetilde{B}(z) .
$$

Thus, matching the coefficients of $z^{u}$ on both sides of (3.26) yields (3.19).

\section{Applications}

In this section, we apply explicit expression (3.19) to derive some important actuarial quantities which are just special cases of $m(u)$ with different choices of $\omega\left(v_{1}, v_{2}\right)$.

\subsection{Ruin probability}

We first consider the ruin probability $\Psi(u)$ defined by

$$
\Psi(u)=P\left(\tau<\infty \mid U_{0}=u\right) .
$$

From (2.4), $m(u)$ reduces to $\Psi(u)$ if $\omega\left(v_{1}, v_{2}\right)=1$. In this case, (3.4)-(3.7) become

$$
\begin{aligned}
& W_{1}(u)=\sum_{i=u+1}^{\infty} h_{i}=\bar{H}(u), \quad \text { with } \quad W_{1}(0)=1, \\
& W_{2}(u)=\sum_{k=u+1}^{\infty} f_{k}=\bar{F}(u), \quad \text { with } \quad W_{2}(0)=1, \\
& W_{3}(u)=\sum_{l=u+1}^{\infty} g_{l}=\bar{G}(u), \quad \text { with } \quad W_{3}(0)=1, \\
& W_{4}(u)=\sum_{j=u+1}^{\infty} \gamma_{j}=\bar{\Gamma}(u), \quad \text { with } \quad W_{4}(0)=1,
\end{aligned}
$$

where

$$
\gamma_{j}=P\left(X_{1}+Y_{1}+Y_{2}=j\right)=f * g^{* 2}(j) .
$$

Similar to (3.14) and (4.6), let

$$
\begin{gathered}
\gamma_{j}^{\prime}=P\left(X_{1}+X_{2}+Y_{1}=j\right)=f^{* 2} * g(j), \\
\pi_{j}=P\left(X_{1}+X_{2}+Y_{1}+Y_{2}=j\right)=f^{* 2} * g^{* 2}(j) .
\end{gathered}
$$


Then, we have, for $\nu=0,1,2$,

$$
\begin{aligned}
& \sum_{j=u}^{\infty} \sum_{i=1}^{j} W_{1}(i) h_{j-i+\nu}=\sum_{j=u}^{\infty} \sum_{i=1}^{j} \bar{H}(i) h_{j-i+\nu}=\sum_{j=u}^{\infty} \sum_{i=\nu}^{j-1+\nu} \bar{H}(j-i+\nu) h_{i} \\
= & \sum_{j=u}^{\infty} P\left(X_{1}+Y_{1}+X_{2}+Y_{2}>j+\nu, \quad X_{2}+Y_{2} \leq j-1+\nu\right) \\
= & \sum_{j=u}^{\infty}\left[P\left(X_{1}+Y_{1}+X_{2}+Y_{2}>j+\nu\right)-P\left(X_{2}+Y_{2}>j-1+\nu\right)\right] \\
= & \sum_{j=u}^{\infty}[\bar{\Pi}(j+\nu)-\bar{H}(j-1+\nu)] .
\end{aligned}
$$

Along the same line, we obtain

$$
\begin{aligned}
& \sum_{j=u}^{\infty} \sum_{k=1}^{j+1} W_{1}(k) f_{j-k+\nu}=\sum_{j=u}^{\infty}\left[\overline{\Gamma^{\prime}}(j+\nu)-\bar{F}(j-1+\nu)\right], \\
& \sum_{j=u}^{\infty} \sum_{i=1}^{j} W_{2}(i) h_{j-i+\nu}=\sum_{j=u}^{\infty}\left[\overline{\Gamma^{\prime}}(j+\nu)-\bar{H}(j-1+\nu)\right] \\
& \sum_{j=u}^{\infty} \sum_{k=1}^{j+1} W_{3}(k) f_{j-k+\nu}=\sum_{j=u}^{\infty}[\bar{H}(j+\nu)-\bar{F}(j-1+\nu)]
\end{aligned}
$$

and

$$
\sum_{j=u}^{\infty} \sum_{k=1}^{j+1} W_{4}(k) f_{j-k+\nu}=\sum_{j=u}^{\infty}[\bar{\Pi}(j+\nu)-\bar{F}(j-1+\nu)]
$$

Substituting (4.2)-(4.5) and (4.7)-(4.11) into (3.18), we get

$$
A^{\Psi}(u)=-q(1-\theta)(1-\alpha)(1-\Psi(0))[(1-\alpha) \bar{H}(u+1)+\alpha \bar{H}(u)]+\sum_{j=u+1}^{\infty} \bar{H}(j)+\alpha \bar{H}(u) .
$$

This together with Theorem 3.1 yields the following corollary.

Corollary 4.1 For every nonnegative initial surplus u, the ruin probability for risk model (2.1) is

$$
\Psi(u)=\frac{p}{1-\alpha-p\left(\mu_{X}+\mu_{Y}\right)} \sum_{j=0}^{u} A^{\Psi}(u-j) \sigma(j),
$$

where $\sigma(j)$ and $A^{\Psi}(u)$ are defined in (3.20) and (4.12), respectively.

When the initial surplus $u=0$,

$$
\Psi(0)=\frac{p}{1-\alpha-p\left(\mu_{X}+\mu_{Y}\right)} A^{\Psi}(0) \sigma(0) .
$$

From (3.20)-(3.22), one can show that

$$
\begin{aligned}
\sigma(0) & =\frac{\lambda}{1+\lambda}+\frac{\lambda}{1+\lambda} \sum_{n=1}^{\infty}\left(\frac{1}{1+\lambda}\right)^{n} \rho^{* n}(0) \\
& =\frac{1-\alpha-p\left(\mu_{X}+\mu_{Y}\right)}{1-q \alpha}\left[1+\sum_{n=1}^{\infty}\left(\frac{p\left(\alpha+\mu_{X}+\mu_{Y}\right)}{1-q \alpha}\right)^{n}\left(\frac{1}{\alpha+\mu_{X}+\mu_{Y}}\right)^{n}\right] \\
& =\frac{1-\alpha-p\left(\mu_{X}+\mu_{Y}\right)}{q(1-\alpha)} .
\end{aligned}
$$


Also, by (4.12), we have

$$
A^{\Psi}(0)=q(1-\theta)(1-\alpha) \Psi(0)+\mu_{X}+\mu_{Y}-(1-\alpha)[1+q(1-\theta)] .
$$

Finally, substituting (4.15) and (4.16) into (4.14) gives

$$
\Psi(0)=\frac{p\left[\mu_{X}+\mu_{Y}-(1-\alpha)[1+q(1-\theta)]\right]}{q(q+p \theta)(1-\alpha)} .
$$

Remark 4.1 If $\alpha=0$,

$$
\Psi(0)=\frac{p\left[\mu_{X}+\mu_{Y}-1-q(1-\theta)\right]}{q(q+p \theta)},
$$

which coincides with (15) of Xiao and Guo (2007). Note that a main claim occurs with probability $q$ rather than $p$ in their paper. And, if $\theta=1$,

$$
\Psi(0)=\frac{p\left[\mu_{X}+\mu_{Y}-(1-\alpha)\right]}{q(1-\alpha)},
$$

which is equivalent to (5.3) of Tan and Yang (2006).

\subsection{Probability function of the deficit at ruin}

Set $\omega\left(v_{1}, v_{2}\right)=\mathbf{1}_{\left(v_{2}=y\right)}$ in $(2.4)$ for $y=1,2, \ldots$ Then,

$$
m(u)=P\left(\left|U_{\tau}\right|=y, \tau<\infty \mid U_{0}=u\right)=f(y \mid u),
$$

which is the probability function of the deficit at ruin. In this case,

$$
\begin{aligned}
& W_{1}(u)=\sum_{i=u+1}^{\infty} \mathbf{1}_{(i-u=y)} h_{i}=h_{u+y}, \quad \text { with } \quad W_{1}(0)=h_{y}, \\
& W_{2}(u)=\sum_{k=u+1}^{\infty} \mathbf{1}_{(k-u=y)} f_{k}=f_{u+y}, \quad \text { with } \quad W_{2}(0)=f_{y}, \\
& W_{3}(u)=\sum_{l=u+1}^{\infty} \mathbf{1}_{(l-u=y)} g_{l}=g_{u+y}, \quad \text { with } \quad W_{3}(0)=g_{y}, \\
& W_{4}(u)=\sum_{j=u+1}^{\infty} \mathbf{1}_{(j-u=y)} \gamma_{j}=\gamma_{u+y}, \quad \text { with } \quad W_{4}(0)=\gamma_{y},
\end{aligned}
$$

and for $\nu=0,1,2$,

$$
\begin{aligned}
\sum_{j=u}^{\infty} \sum_{i=1}^{j} W_{1}(i) h_{j-i+\nu} & =\bar{H}(y)-\sum_{i=1}^{u-1} h_{i+y} H(u-i-1+\nu), \\
\sum_{j=u}^{\infty} \sum_{k=1}^{j+1} W_{1}(k) f_{j-k+\nu} & =\bar{H}(y)-\sum_{k=1}^{u} h_{k+y} F(u-k-1+\nu), \\
\sum_{j=u}^{\infty} \sum_{i=1}^{j} W_{2}(i) h_{j-i+\nu} & =\bar{F}(y)-\sum_{i=1}^{u-1} f_{i+y} H(u-i-1+\nu),
\end{aligned}
$$




$$
\begin{aligned}
& \sum_{j=u}^{\infty} \sum_{k=1}^{j+1} W_{3}(k) f_{j-k+\nu}=\bar{G}(y)-\sum_{k=1}^{u} g_{k+y} F(u-k-1+\nu), \\
& \sum_{j=u}^{\infty} \sum_{k=1}^{j+1} W_{4}(k) f_{j-k+\nu}=\bar{\Gamma}(y)-\sum_{k=1}^{u} \gamma_{k+y} F(u-k-1+\nu) .
\end{aligned}
$$

By inserting (4.18)-(4.26) into (3.18), we have

$$
\begin{aligned}
& A^{f(y \mid u)}(u) \\
= & q(1-\theta)(1-\alpha) f(y \mid 0)[(1-\alpha) \bar{H}(u+1)+\alpha \bar{H}(u)] \\
+ & {[\theta \bar{H}(u+y)+(1-\theta) \bar{F}(u+y)]+\alpha\left[\theta h_{u+y}+(1-\theta) f_{u+y}\right] } \\
- & p(1-\theta) \alpha\left[\theta h_{y}+(1-\theta) f_{y}\right][(1-\alpha) \bar{H}(u)+\alpha \bar{H}(u-1)] \\
+ & (1-\theta) \alpha\left[p(1-\theta) h_{y}+q g_{y}+p \theta \gamma_{y}\right][(1-\alpha) \bar{F}(u)+\alpha \bar{F}(u-1)] \\
- & p(1-\theta)[\theta \bar{H}(y)+(1-\theta) \bar{F}(y)]+(1-\theta)[p(1-\theta) \bar{H}(y)+q \bar{G}(y)+p \theta \bar{\Gamma}(y)] \\
+ & p(1-\theta) \sum_{i=1}^{u-1}\left[\theta h_{i+y}+(1-\theta) f_{i+y}\right]\left[(1-\alpha)^{2} H(u-i+1)+2 \alpha(1-\alpha) H(u-i)+\alpha^{2} H(u-i-1)\right] \\
- & (1-\theta) \sum_{k=1}^{u}\left[p(1-\theta) h_{k+y}+q g_{k+y}+p \theta \gamma_{k+y}\right]\left[(1-\alpha)^{2} F(u-k+1)+2 \alpha(1-\alpha) F(u-k)+\alpha^{2} F(u-k-1)\right] .
\end{aligned}
$$

So, we have

Corollary 4.2 For risk model (2.1), the probability function of the deficit at ruin is

$$
f(y \mid u)=\frac{p}{1-\alpha-p\left(\mu_{X}+\mu_{Y}\right)} \sum_{j=0}^{u} A^{f(y \mid u)}(u-j) \sigma(j), \quad y=1,2, \ldots,
$$

where $\sigma(j)$ and $A^{f(y \mid u)}(u)$ are defined in (3.20) and (4.27), respectively.

In particular,

$$
\begin{aligned}
A^{f(y \mid 0)}(0)= & q(1-\theta)(1-\alpha) f(y \mid 0) \\
& +(q+p \theta)[\theta \bar{H}(y)+(1-\theta) \bar{F}(y)]+(1-\theta)[p(1-\theta) \bar{H}(y)+q \bar{G}(y)+p \theta \bar{\Gamma}(y)] \\
& +(q+p \theta) \alpha\left[\theta h_{y}+(1-\theta) f_{y}\right]+(1-\theta) \alpha\left[p(1-\theta) h_{y}+q g_{y}+p \theta \gamma_{y}\right] .
\end{aligned}
$$

Combining (4.29) with (4.15) and (4.28), we obtain

$$
f(y \mid 0)=\frac{\left\{\begin{array}{l}
p(q+p \theta)[\theta \bar{H}(y)+(1-\theta) \bar{F}(y)]+p(1-\theta)[p(1-\theta) \bar{H}(y)+q \bar{G}(y)+p \theta \bar{\Gamma}(y)] \\
+p(q+p \theta) \alpha\left[\theta h_{y}+(1-\theta) f_{y}\right]+p(1-\theta) \alpha\left[p(1-\theta) h_{y}+q g_{y}+p \theta \gamma_{y}\right]
\end{array}\right\}}{q(q+p \theta)(1-\alpha)} .
$$

Remark 4.2 If $\theta=1$ in (4.30), then

$$
f(y \mid 0)=\frac{p\left[\bar{H}(y)+\alpha h_{y}\right]}{q(1-\alpha)},
$$


and the corresponding distribution function is given by

$$
F(y \mid 0)=\frac{p}{q(1-\alpha)} \sum_{j=1}^{y}\left[\bar{H}(j)+\alpha h_{j}\right],
$$

which is equivalent to (5.9) of Tan and Yang (2006).

\subsection{Joint probability function of the surplus immediately before ruin and the deficit at ruin}

Now, let $\omega\left(v_{1}, v_{2}\right)=\mathbf{1}_{\left(v_{1}=x, v_{2}=y\right)}$ in $(2.4)$ for $x=0,1, \ldots$ and $y=1,2, \ldots$ Then,

$$
m(u)=P\left(U_{\tau-}=x,\left|U_{\tau}\right|=y, \tau<\infty \mid U_{0}=u\right)=f(x, y \mid u),
$$

which is the joint probability function of the surplus immediately before ruin and the deficit at ruin. In this case,

$$
\begin{aligned}
& W_{1}(u)=\sum_{i=u+1}^{\infty} \mathbf{1}_{(u=x, i-u=y)} h_{i}=\mathbf{1}_{(u=x)} h_{x+y}, \quad \text { with } \quad W_{1}(0)=\mathbf{1}_{(x=0)} h_{y}, \\
& W_{2}(u)=\sum_{k=u+1}^{\infty} \mathbf{1}_{(u=x, k-u=y)} f_{k}=\mathbf{1}_{(u=x)} f_{x+y}, \quad \text { with } \quad W_{2}(0)=\mathbf{1}_{(x=0)} f_{y}, \\
& W_{3}(u)=\sum_{l=u+1}^{\infty} \mathbf{1}_{(u=x, l-u=y)} g_{l}=\mathbf{1}_{(u=x)} g_{x+y}, \quad \text { with } \quad W_{3}(0)=\mathbf{1}_{(x=0)} g_{y}, \\
& W_{4}(u)=\sum_{j=u+1}^{\infty} \mathbf{1}_{(u=x, j-u=y)} \gamma_{j}=\mathbf{1}_{(u=x)} \gamma_{x+y}, \quad \text { with } \quad W_{4}(0)=\mathbf{1}_{(x=0)} \gamma_{y},
\end{aligned}
$$

and for $\nu=0,1,2$,

$$
\begin{aligned}
& \sum_{j=u}^{\infty} \sum_{i=1}^{j} W_{1}(i) h_{j-i+\nu}=\mathbf{1}_{(x \geq 1)}\left[\mathbf{1}_{(u \leq x)} h_{x+y}+\mathbf{1}_{(u \geq x+1)} h_{x+y} \bar{H}(u-x-1+\nu)\right], \\
& \sum_{j=u}^{\infty} \sum_{k=1}^{j+1} W_{1}(k) f_{j-k+\nu}=\mathbf{1}_{(x \geq 1)}\left[\mathbf{1}_{(u \leq x-1)} h_{x+y}+\mathbf{1}_{(u \geq x)} h_{x+y} \bar{F}(u-x-1+\nu)\right], \\
& \sum_{j=u}^{\infty} \sum_{i=1}^{j} W_{2}(i) h_{j-i+\nu}=\mathbf{1}_{(x \geq 1)}\left[\mathbf{1}_{(u \leq x)} f_{x+y}+\mathbf{1}_{(u \geq x+1)} f_{x+y} \bar{H}(u-x-1+\nu)\right], \\
& \sum_{j=u}^{\infty} \sum_{k=1}^{j+1} W_{3}(k) f_{j-k+\nu}=\mathbf{1}_{(x \geq 1)}\left[\mathbf{1}_{(u \leq x-1)} g_{x+y}+\mathbf{1}_{(u \geq x)} g_{x+y} \bar{F}(u-x-1+\nu)\right], \\
& \sum_{j=u}^{\infty} \sum_{k=1}^{j+1} W_{4}(k) f_{j-k+\nu}=\mathbf{1}_{(x \geq 1)}\left[\mathbf{1}_{(u \leq x-1)} \gamma_{x+y}+\mathbf{1}_{(u \geq x)} \gamma_{x+y} \bar{F}(u-x-1+\nu)\right] .
\end{aligned}
$$


Inserting (4.31)-(4.39) into (3.18) yields

$$
\begin{aligned}
& f(x, y \mid u) \\
&= q(1-\theta)(1-\alpha) f(x, y \mid 0)[(1-\alpha) \bar{H}(u+1)+\alpha \bar{H}(u)]+\left[\theta h_{x+y}+(1-\theta) f_{x+y}\right]\left[\mathbf{1}_{(u \leq x-1)}+\alpha \mathbf{1}_{(u=x)}\right] \\
&-p(1-\theta) \alpha \mathbf{1}_{(x=0)}\left[\theta h_{y}+(1-\theta) f_{y}\right][(1-\alpha) \bar{H}(u)+\alpha \bar{H}(u-1)] \\
&+(1-\theta) \alpha \mathbf{1}_{(x=0)}\left[p(1-\theta) h_{y}+q g_{y}+p \theta \gamma_{y}\right][(1-\alpha) \bar{F}(u)+\alpha \bar{F}(u-1)] \\
&- p(1-\theta) \mathbf{1}_{(x \geq 1)}\left[\theta h_{x+y}+(1-\theta) f_{x+y}\right]\left[\mathbf{1}_{(u \geq x+1)} \bar{H}(u-x+1)+\mathbf{1}_{(u \leq x)}\right. \\
&\left.+\alpha \mathbf{1}_{(u \geq x+1)}\left((2-\alpha) h_{u-x+1}+\alpha h_{u-x}\right)\right] \\
&+(1-\theta) \mathbf{1}_{(x \geq 1)}\left[p(1-\theta) h_{x+y}+q g_{x+y}+p \theta \gamma_{x+y}\right]\left[\mathbf{1}_{(u \geq x)} \bar{F}(u-x+1)+\mathbf{1}_{(u \leq x-1)}\right. \\
&\left.\quad+\alpha \mathbf{1}_{(u \geq x)}\left((2-\alpha) f_{u-x+1}+\alpha f_{u-x}\right)\right] .
\end{aligned}
$$

Thus, we have

Corollary 4.3 For risk model (2.1), the joint probability function of the surplus immediately before ruin and the deficit at ruin is

$$
f(x, y \mid u)=\frac{p}{1-\alpha-p\left(\mu_{X}+\mu_{Y}\right)} \sum_{j=0}^{u} A^{f(x, y \mid u)}(u-j) \sigma(j), \quad x=0,1, \ldots, y=1,2, \ldots,
$$

where $\sigma(j)$ and $A^{f(x, y \mid u)}(u)$ are defined in (3.20) and (4.40), respectively.

For $u=0$,

$$
\begin{aligned}
A^{f(x, y \mid 0)}(0)= & q(1-\theta)(1-\alpha) f(x, y \mid 0) \\
& +\mathbf{1}_{(x \geq 1)}\left[(q+p \theta)\left[\theta h_{x+y}+(1-\theta) f_{x+y}\right]+(1-\theta)\left[p(1-\theta) h_{x+y}+q g_{x+y}+p \theta \gamma_{x+y}\right]\right] \\
& +\alpha \mathbf{1}_{(x=0)}\left[(q+p \theta)\left[\theta h_{y}+(1-\theta) f_{y}\right]+(1-\theta)\left[p(1-\theta) h_{y}+q g_{y}+p \theta \gamma_{y}\right]\right] .
\end{aligned}
$$

Combining (4.42) with (4.15) and (4.41) leads to

$$
f(x, y \mid 0)=\frac{\left\{\begin{array}{l}
p \mathbf{1}_{(x \geq 1)}\left[(q+p \theta)\left[\theta h_{x+y}+(1-\theta) f_{x+y}\right]+(1-\theta)\left[p(1-\theta) h_{x+y}+q g_{x+y}+p \theta \gamma_{x+y}\right]\right] \\
+p \alpha \mathbf{1}_{(x=0)}\left[(q+p \theta)\left[\theta h_{y}+(1-\theta) f_{y}\right]+(1-\theta)\left[p(1-\theta) h_{y}+q g_{y}+p \theta \gamma_{y}\right]\right]
\end{array}\right\}}{q(q+p \theta)(1-\alpha)} .
$$

Remark 4.3 If $\alpha=0$ in (4.43),

$$
f(x, y \mid 0)=\frac{p \mathbf{1}_{(x \geq 1)}\left[(q+p \theta)\left[\theta h_{x+y}+(1-\theta) f_{x+y}\right]+(1-\theta)\left[p(1-\theta) h_{x+y}+q g_{x+y}+p \theta \gamma_{x+y}\right]\right]}{q(q+p \theta)}
$$

which is equivalent to (14) of Xiao and Guo (2007).

Remark 4.4 (4.30) can also be derived by summing $x$ from 0 to $\infty$ in (4.43). 


\subsection{Probability function of the claim causing ruin}

Finally, consider $\omega\left(v_{1}, v_{2}\right)=\mathbf{1}_{\left(v_{1}+v_{2}=s\right)}$ in $(2.4)$ for $s=1,2, \ldots$ Then,

$$
m(u)=P\left(U_{\tau-}+\left|U_{\tau}\right|=s, \tau<\infty \mid U_{0}=u\right)=f_{*}(s \mid u),
$$

which is the probability function of the claim causing ruin. In this case,

$$
\begin{aligned}
& W_{1}(u)=\sum_{i=u+1}^{\infty} \mathbf{1}_{(i=s)} h_{i}=\mathbf{1}_{(u \leq s-1)} h_{s}, \quad \text { with } \quad W_{1}(0)=h_{s}, \\
& W_{2}(u)=\sum_{k=u+1}^{\infty} \mathbf{1}_{(k=s)} f_{k}=\mathbf{1}_{(u \leq s-1)} f_{s}, \quad \text { with } \quad W_{2}(0)=f_{s}, \\
& W_{3}(u)=\sum_{l=u+1}^{\infty} \mathbf{1}_{(l=s)} g_{l}=\mathbf{1}_{(u \leq s-1)} g_{s}, \quad \text { with } \quad W_{3}(0)=g_{s}, \\
& W_{4}(u)=\sum_{j=u+1}^{\infty} \mathbf{1}_{(j=s)} \gamma_{j}=\mathbf{1}_{(u \leq s-1)} \gamma_{s}, \quad \text { with } \quad W_{4}(0)=\gamma_{s},
\end{aligned}
$$

and for $\nu=0,1,2$,

$$
\begin{aligned}
& \sum_{j=u}^{\infty} \sum_{i=1}^{j} W_{1}(i) h_{j-i+\nu}=\mathbf{1}_{(s \geq 2)} h_{s}\left[\mathbf{1}_{(u \leq s-1)}\left(\mu_{X}+\mu_{Y}+1-\nu\right)+\mathbf{1}_{(u \geq s)} \sum_{j=u}^{\infty} \bar{H}(j-s+\nu)\right. \\
& \left.+\mathbf{1}_{(u \leq s-2)}(s-u-1)-\sum_{j=u}^{\infty} \bar{H}(j-1+\nu)\right], \\
& \sum_{j=u}^{\infty} \sum_{k=1}^{j+1} W_{1}(k) f_{j-k+\nu}=\mathbf{1}_{(s \geq 2)} h_{s}\left[\mathbf{1}_{(u \leq s-2)}\left(\mu_{X}+2-\nu\right)+\mathbf{1}_{(u \geq s-1)} \sum_{j=u}^{\infty} \bar{F}(j-s+\nu)\right. \\
& \left.+\mathbf{1}_{(u \leq s-3)}(s-u-2)-\sum_{j=u}^{\infty} \bar{F}(j-1+\nu)\right], \\
& \sum_{j=u}^{\infty} \sum_{i=1}^{j} W_{2}(i) h_{j-i+\nu}=\mathbf{1}_{(s \geq 2)} f_{s}\left[\mathbf{1}_{(u \leq s-1)}\left(\mu_{X}+\mu_{Y}+1-\nu\right)+\mathbf{1}_{(u \geq s)} \sum_{j=u}^{\infty} \bar{H}(j-s+\nu)\right. \\
& \left.+\mathbf{1}_{(u \leq s-2)}(s-u-1)-\sum_{j=u}^{\infty} \bar{H}(j-1+\nu)\right], \\
& \sum_{j=u}^{\infty} \sum_{k=1}^{j+1} W_{3}(k) f_{j-k+\nu}=\mathbf{1}_{(s \geq 2)} g_{s}\left[\mathbf{1}_{(u \leq s-2)}\left(\mu_{X}+2-\nu\right)+\mathbf{1}_{(u \geq s-1)} \sum_{j=u}^{\infty} \bar{F}(j-s+\nu)\right. \\
& \left.+\mathbf{1}_{(u \leq s-3)}(s-u-2)-\sum_{j=u}^{\infty} \bar{F}(j-1+\nu)\right], \\
& \sum_{j=u}^{\infty} \sum_{k=1}^{j+1} W_{4}(k) f_{j-k+\nu}=\mathbf{1}_{(s \geq 2)} \gamma_{s}\left[\mathbf{1}_{(u \leq s-2)}\left(\mu_{X}+2-\nu\right)+\mathbf{1}_{(u \geq s-1)} \sum_{j=u}^{\infty} \bar{F}(j-s+\nu)\right. \\
& \left.+\mathbf{1}_{(u \leq s-3)}(s-u-2)-\sum_{j=u}^{\infty} \bar{F}(j-1+\nu)\right] .
\end{aligned}
$$


Inserting (4.44)-(4.52) into (3.18), we get

$$
\begin{aligned}
& A^{f_{*}(s \mid u)}(u) \\
& =q(1-\theta)(1-\alpha) f_{*}(s \mid 0)[(1-\alpha) \bar{H}(u+1)+\alpha \bar{H}(u)] \\
& +\left[\theta h_{s}+(1-\theta) f_{s}\right]\left[(1-\alpha) \mathbf{1}_{(u \leq s-2)}(s-u-1)+\alpha \mathbf{1}_{(u \leq s-1)}(s-u)\right] \\
& -p(1-\theta) \alpha\left[\theta h_{s}+(1-\theta) f_{s}\right][(1-\alpha) \bar{H}(u)+\alpha \bar{H}(u-1)] \\
& +(1-\theta) \alpha\left[p(1-\theta) h_{s}+q g_{s}+p \theta \gamma_{s}\right][(1-\alpha) \bar{F}(u)+\alpha \bar{F}(u-1)] \\
& -p(1-\theta) \mathbf{1}_{(s \geq 2)}\left[\theta h_{s}+(1-\theta) f_{s}\right]\left\{\left[\mathbf{1}_{(u \leq s-1)}\left(\mu_{X}+\mu_{Y}-1\right)+\mathbf{1}_{(u \geq s)} \sum_{j=u}^{\infty} \bar{H}(j-s+2)\right.\right. \\
& \left.+\mathbf{1}_{(u \leq s-2)}(s-u-1)-\sum_{j=u}^{\infty} \bar{H}(j+1)\right] \\
& +2 \alpha \mathbf{1}_{(u \leq s-1)}+\alpha(2-\alpha)\left[\mathbf{1}_{(u \geq s)} \bar{H}(u-s+1)-\bar{H}(u)\right] \\
& \left.+\alpha^{2}\left[\mathbf{1}_{(u \geq s)} \bar{H}(u-s)-\bar{H}(u-1)\right]\right\} \\
& +(1-\theta) \mathbf{1}_{(s \geq 2)}\left[p(1-\theta) h_{s}+q g_{s}+p \theta \gamma_{s}\right]\left\{\left[\mathbf{1}_{(u \leq s-2)} \mu_{X}+\mathbf{1}_{(u \geq s-1)} \sum_{j=u}^{\infty} \bar{F}(j-s+2)\right.\right. \\
& \left.+\mathbf{1}_{(u \leq s-3)}(s-u-2)-\sum_{j=u}^{\infty} \bar{F}(j+1)\right] \\
& +2 \alpha \mathbf{1}_{(u \leq s-2)}+\alpha(2-\alpha)\left[\mathbf{1}_{(u \geq s-1)} \bar{F}(u-s+1)-\bar{F}(u)\right] \\
& \left.+\alpha^{2}\left[\mathbf{1}_{(u \geq s-1)} \bar{F}(u-s)-\bar{F}(u-1)\right]\right\} \text {. }
\end{aligned}
$$

Therefore, we have the following result.

Corollary 4.4 For risk model (2.1), the probability function of the claim causing ruin satisfies

$$
f_{*}(s \mid u)=\frac{p}{1-\alpha-p\left(\mu_{X}+\mu_{Y}\right)} \sum_{j=0}^{u} A^{f_{*}(s \mid u)}(u-j) \sigma(j), \quad s=1,2, \ldots,
$$

where $\sigma(j)$ and $A^{f_{*}(s \mid u)}(u)$ are defined in (3.20) and (4.53), respectively.

Again, for $u=0$,

$$
\begin{aligned}
A_{*(s \mid 0)}^{f_{*}(0)=} & q(1-\theta)(1-\alpha) f_{*}(s \mid 0) \\
& +(s-1+\alpha)\left[(q+p \theta)\left[\theta h_{s}+(1-\theta) f_{s}\right]+(1-\theta)\left[p(1-\theta) h_{s}+q g_{s}+p \theta \gamma_{s}\right]\right] .
\end{aligned}
$$

Combining (4.55) with (4.15) and (4.54), we have

$$
f_{*}(s \mid 0)=\frac{(s-1+\alpha) p\left[(q+p \theta)\left[\theta h_{s}+(1-\theta) f_{s}\right]+(1-\theta)\left[p(1-\theta) h_{s}+q g_{s}+p \theta \gamma_{s}\right]\right]}{q(q+p \theta)(1-\alpha)} .
$$

Remark 4.5 If $\theta=1$ and $\alpha=0$ in (4.56), then $f_{*}(s \mid 0)=(p / q)(s-1) h_{s}$ which is equivalent to the last relation in Pavlova and Willmot (2004). 
Remark 4.6 All the formulae for the case of zero initial surplus, including (4.17), (4.30), (4.43) and (4.56), can also be obtained directly from (3.13). The reason why we utilize (3.19) is to illustrate the usage of Theorem 3.1.

\section{Acknowledgements}

The research of Kam Chuen Yuen was supported by a university research grant of the University of Hong Kong. The research of Jinzhu Li and Rong Wu was supported by National Basic Research Program of China (973 Program) 2007CB814905.

\section{References}

[1] Gerber, H.U., 1988. Mathematical fun with the compound binomial process. ASTIN Bulletin 18, 161168.

[2] Gerber, H.U., Shiu, E.S.W., 1998. On the time value of ruin. North American Actuarial Journal 2, 48-78.

[3] Pavlova, K.P., Willmot, G.E., 2004. The discrete stationary renewal risk model and the Gerber-Shiu discounted penalty function. Insurance: Mathematics and Economics 35, 267-277.

[4] Shiu, E.S.W., 1989. The probability of eventual ruin in a compound binomial model. ASTIN Bulletin 19, 179-190.

[5] Tan, J., Yang, X., 2006. The compound binomial model with randomized decisions on paying dividends. Insurance Mathematics and Economics 39, 1-18.

[6] Willmot, G. E., 1993. Ruin probabilities in the compound binomial model. Insurance Mathematics and Economics 12, 133-142.

[7] Xiao, Y., Guo, J., 2007. The compound binomial risk model with time-correlated claims. Insurance: Mathematics and Economics 41, 124-133.

[8] Yuen, K.C., Guo, J., 2001. Ruin probabilities for time-correlated claims in the compound binomial model. Insurance: Mathematics and Economics 29, 47-57.

[9] Yuen, K.C., Guo, J. and Ng, K.W., 2005. Ultimate ruin in a delayed-claims risk model. Journal of Applied Probability 42, 163-174. 